\title{
microRNAs in a multicellular green alga Volvox carteri
}

\author{
LI JingRui ${ }^{1,2,3 \dagger}$, WU Yang ${ }^{1,2,3 \dagger}$ \& QI YiJun ${ }^{1,2,3^{*}}$ \\ ${ }^{1}$ Center for Plant Biology, School of Life Sciences, Tsinghua University, Beijing 100084, China; \\ ${ }^{2}$ Tsinghua-Peking Center for Life Sciences, Beijing 100084, China; \\ ${ }^{3}$ School of Life Science, Tsinghua University, Beijing 100084, China
}

Received October 15, 2013; accepted November 20, 2013; published online December 23, 2013

\begin{abstract}
microRNAs (miRNAs) have emerged as key components in the eukaryotic gene regulatory network. We and others have previously identified many miRNAs in a unicellular green alga, Chlamydomonas reinhardtii. To investigate whether miRNA-mediated gene regulation is a general mechanism in green algae and how miRNAs have been evolved in the green algal lineage, we examined small RNAs in Volvox carteri, a multicellular species in the same family with Chlamydomonas reinhardtii. We identified 174 miRNAs in Volvox, with many of them being highly enriched in gonidia or somatic cells. The targets of the miRNAs were predicted and many of them were subjected to miRNA-mediated cleavage in vivo, suggesting that miRNAs play regulatory roles in the biology of green algae. Our catalog of miRNAs and their targets provides a resource for further studies on the evolution, biological functions, and genomic properties of miRNAs in green algae.
\end{abstract}

Volvox, small RNA, microRNA, conservation, evolution

Citation: $\quad$ Li JR, Wu Y, Qi YJ. microRNAs in a multicellular green alga Volvox carter. Sci China Life Sci, 2014, 57: 36-45, doi: 10.1007/s11427-013-4580-3

microRNAs (miRNAs) are small non-coding RNAs that play important roles in gene regulation in eukaryotes $[1,2]$. miRNAs are processed from their stem-loop structured precursors (pre-miRNAs) by Dicer or Dicer-like proteins $[3,4]$ and incorporated into effector complexes that contain Argonaute (AGO) family proteins. miRNAs regulate target gene expression through mRNA degradation or translation inhibition. The mRNA degradation is achieved through miRNA-directed cleavage in plants and deadenylation followed by mRNA decay in animals $[1,2]$.

We and others have previously found many miRNAs in a single-celled green alga Chlamydomonas reinhardtii $[5,6]$, suggesting that the miRNA pathway is an ancient mechanism of gene regulation that has evolved prior to the emergence of multicellularity. Intriguingly, no universally conserved miRNAs have been found among plants, animals and

$\dagger$ Contributed equally to this work

*Corresponding author (email: qiyijun@ tsinghua.edu.cn)
Chlamydomonas [5,6]. This suggests that miRNAs may have evolved independently in the lineages leading to animals, plants, and green algae.

Chlamydomonas belongs to a conspicuous green algae lineage Volvocine, which represents an excellent model system for exploring the events during evolutionary transition from unicellular to multicellular organisms [7]. Volvox carteri, the most evolved species in Volvocine, diverged from unicellular ancestor nearly 200 million years ago and has similar protein coding potentials compared to Chlamydomonas [8-10]. One Volvox spherical colony consists of 2000 cells: 16 large reproductive cells (called gonidia) and thousands of small motile somatic cells $[9,11]$.

To investigate whether miRNA-mediated gene regulation is a general mechanism in green algae and how miRNAs have been evolved in the green algal lineage, we examined miRNAs in Volvox. We profiled small RNAs in Volvox spherical colonies, gonidia, and somatic cells, and annotated 174 miRNAs. We found some miRNAs are enriched in go- 
nidia or in somatic cells, suggesting potential regulatory roles for miRNAs in specifying cell types. We predicted miRNA target genes and detected miRNA-mediated cleavage of some targets. Intriguingly, only one miRNA was found to be conserved in Chlamydomonas and Volvox, suggesting the high frequency of birth and death of miRNAs in green algae.

\section{Materials and methods}

\subsection{Strain and culture conditions}

Volvox carteri (NIES-865) was obtained from National Institute for Environmental Studies (NIES) and cultured in MG liquid medium (http://mcc.nies.go.jp/) at $23^{\circ} \mathrm{C}, 48 \mu \mathrm{E}$ $\mathrm{m}^{-2} \mathrm{~s}^{-1}$.

\subsection{Small RNA preparation and cloning}

Gonidia and somatic cells were purified using an established method [12]. Total RNAs were extracted from Volvox whole colonies, gonidia and somatic cells using TRIzol reagent (Invitrogen, Carlsbad, USA). Small RNAs were isolated and cDNA libraries were constructed as described [5]. The libraries were sequenced on an Illumina GAIIx sequencing platform.

\subsection{Processing of the Volvox genome sequence}

The Volvox genome (version 2.0) was downloaded from the JGI Volvox website (http://genome.jgi-psf.org/Volca1/ Volca1.download.ftp.html). To facilitate data analysis, we linked downloaded scaffolds to build an arbitrary genome. All analyses described in the text were carried out using information extracted from this arbitrary genome. Repeat regions and transposable elements of the Volvox genome were identified by Repeat Masker (v3.1.3) according to RepBase (v10.09) [13].

\subsection{Small RNA analysis}

Low-quality reads generated from Illumina GAIIx were discarded and the rest were collected and the adaptor sequences were removed. The small RNAs with a length of 19-27 nt were mapped to the Volvox genome sequences. The small RNAs with perfect genomic matches were used for further analysis. The relative frequency of each nucleotide at each position of the small RNAs was calculated and graphically represented using Weblogo [14]. Relationships between small RNAs and annotated genes were determined by comparing the genomic loci of small RNAs with the BLAST results of gene sequences. Small RNAs derived from known non-coding RNAs were identified by comparing small RNAs with the sequences of non-coding RNAs collected in Rfam (http://www.sanger.ac.uk/Software/Rfam/) [15].

\subsection{Identification of Volvox miRNAs and target genes}

The prediction of Volvox miRNAs and their targets was carried out as previously described [5].

\subsection{Quantitative RT-PCR analysis}

Total RNAs were extracted from whole colonies, gonidia and somatic cells of Volvox by using TRIzol reagent (Invitrogen). Contaminated DNAs were removed by digestion with RNase-free DNase (Promega, Fitchburg, USA). RNAs were reverse-transcribed by M-MLV (Promega). Quantitative PCR was performed using SYBR Premix EX Taq (TaKaRa, Dalian, China) on Mastercyclerep-realplex (Eppendorf, Hamburg, Germany). The sequences of primers are listed in Table S1 in Supporting Information.

\subsection{Small RNA Northern blot}

Small RNA for Northern blot analysis was prepared as described [5]. ${ }^{32} \mathrm{P}$ end-labeled oligo-nucleotide probes were used. The sequences of the probes are listed in Table S1 in Supporting Information.

\subsection{Degradome library construction and data pro- cessing}

Degradome libraries were constructed as described [16,17]. After discarding low-quality reads and removing adaptor sequences, signatures were mapped to genome sequences and mapped reads were treated as dataset for Cleaveland [18].

\subsection{Accession numbers}

Small RNA datasets used here were deposited in the Gene Expression Omnibus (GEO) repository (http://www.ncbi. nlm.nih.gov/geo/) under the following accession number: GSE52345.

\section{Results and discussion}

\subsection{Small RNAs in Volvox}

To profile small RNAs in Volvox, we purified gonidia and somatic cells using an established method [12]. The quality of purification was examined by microscopic observation (Figure 1A) and the detection of gonidia and somatic cellspecific genes (Figure 1B). Small RNAs of 19-28 nt were prepared from Volvox spherical colonies, gonidia, and somatic cells for construction of cDNA libraries. The libraries were subjected to Illumina GAIIx sequencing platform.

Sequencing reads are mapped to the genome of Volvox (http://genome.jgi-psf.org/Volca1/Volca1.download.ftp.html). In total, 3316336, 3619150, and 3925000 reads were 

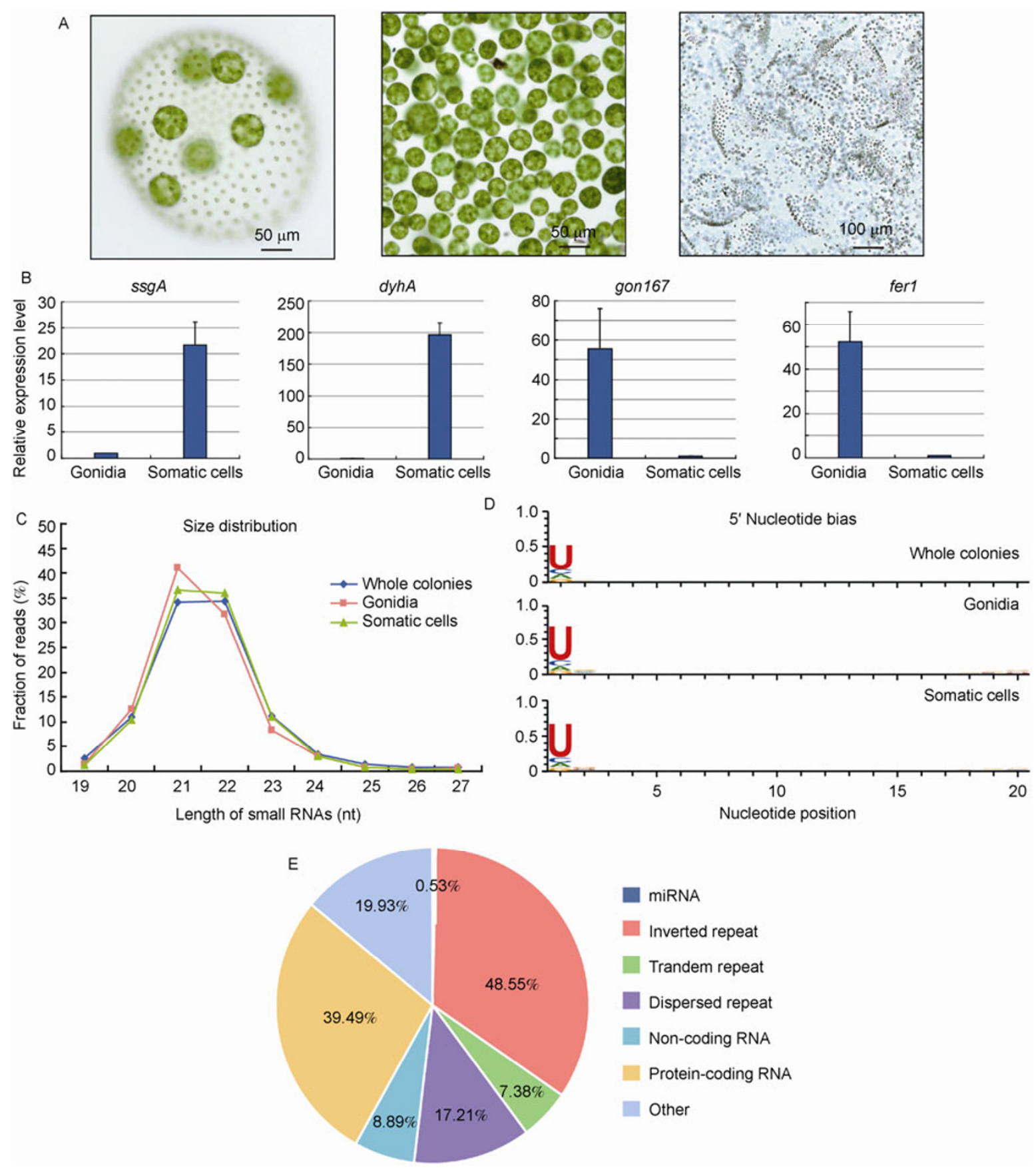

Figure 1 Small RNAs in Volvox. A, Microscopic photographs of adult Volvox (Volvox carteri f. nagariensis lyengar, NIES-865) spherical colony (left) and gonidia (middle) and somatic cells (right). B, Detection of gonidia- and somatic-cell-specific genes in gonidia and somatic cells using quantitative RT-PCR. $\mathrm{C}$, Size distribution of Volvox small RNAs in whole colonies (blue), gonidia (red) and somatic cells (green). D, The relative nucleotide bias at each position of the small RNAs in whole colonies, gonidia and somatic cells. The graphics were made by using Weblogo [23]. The sequence conservation at each position is indicated by the overall height of the stack of symbols (U, A, C and G), while the relative frequency of each nucleotide is represented by the height of the corresponding symbol. E, A pie chart summarizing the annotation of small RNA populations in Volvox whole colonies.

obtained for the spheroids, gonidia, somatic cells, respectively, which represent 317362,221582 , and 248279 unique small RNA sequences (Table S2 in Supporting Information). Further analyses showed that small RNAs in Volvox are predominantly 21-22 nt in length (Figure 1C) and exhibit an overwhelming $5^{\prime} \mathrm{U}$ bias (Figure 1D). Intriguingly, about $2 / 3$ of the small RNAs are produced from repeats, especially from inverted repeats (Figure 1E; Table S2 in Supporting Information). The function of these repeat-derived small RNAs remains to be investigated.

\subsection{Identification of miRNAs in Volvox}

We used bioinformatics pipeline that was previously devel- 
oped for annotating Chlamydomonas miRNAs to identify miRNAs in Volvox [5]. A total of 174 miRNAs that belong to 130 families were annotated (Table S3, Dataset S1 in Supporting Information). We found that while some miRNAs are located in exons or introns of annotated Volvox genes, the vast majority of miRNAs are derived from the intergenic regions, reminiscent of those in land plants [4]. Similar to their counterparts in Chlamydomonas, miRNA precursors in Volvox are various in lengths (Figure 2A-C). Interestingly, mature miRNAs within the same family are located in the same arm of the precursors (Figure 2B), which may suggest that they are evolved from a common ancestor. We also found many precursors that can produce more than one mature miRNAs (Figure 2C). For example, Vca-miR14.1 and Vca-miR14.2 are produced from the 5' arm of one precursor, while Vca-miR30-5p and VcamiR30-3p are processed from different arms (Figure 2C; Dataset S1 in Supporting Information).

To confirm the expression of the annotated miRNAs, we performed Northern analysis with small RNAs prepared from Volvox whole colonies. Sixteen Northern confirmed miRNAs were shown (Figure 2D). For some miRNAs (Vca-miR2, Vca-miR14.1, and Vca-miR18), more than one band were detected, which is consistent with the deep sequencing data (Dataset S1 in Supporting Information).

\subsection{Prediction and verification of miRNA targets in Volvox}

We and others have previously shown that Chlamydomonas miRNAs have extensive sequence complementarities to their target genes and can mediate the cleavage of the target mRNAs [5,6]. To investigate whether this holds true in Volvox, we predicted the target genes of Volvox miRNAs using a bioinformatics method that we previously developed for predicting Chlamydomonas miRNA targets. Two hundred and forty-three targets were predicted for 60 miRNA families (Table S4 in Supporting Information). These predicted target genes encode proteins that are involved in various biological pathways (Table S4 in Supporting Information).

Next, we employed a high-throughput "degradome" sequencing approach $[16,17]$ to validate the predicted miRNA targets. We constructed the cDNA library for degradome sequencing as described [16]. High-throughput sequencing resulted in 6005274 reads that match to the transcriptome of Volvox (http://genome.jgi-psf.org/Volca1/Volca1.download. ftp.html). Using Cleaveland, a pipeline for using degradome data to find small RNA targets [18], we found that among 243 predicted targets, 146 had at least one degradome tag with a $5^{\prime}$ end that was precisely opposite the 10th nucleotide of a miRNA, a typical feature of miRNA-directed cleavage (Figure 3; Figure S1-S4 in Supporting Information).

These data indicate that Volvox miRNAs can regulate their targets through mRNA cleavage. However, in light of the fact that plant miRNAs can also regulate target genes through translation repression while they mediate mRNA cleavage [2], it still remains possible that Volvox miRNAs could also mediate translation repression. Our data also suggest that miRNA-mediated mRNA cleavage is probably a conserved mechanism of gene regulation in green algae.

\subsection{Differential expression of miRNAs in gonidia and somatic cells}

In plants and animals, some miRNAs are involved in cellular differentiation $[2,19,20]$. We have previously shown that the expressions of some Chlamydomonas miRNAs are regulated during gametogenesis [5]. As an initial step towards the understanding of the biological function of Volvox miRNAs, we investigated whether miRNAs are differentially expressed in gonidia and somatic cells. The expression levels of the miRNAs were calculated as RPMs (reads per million) in two cell types. We found that 49 miRNAs are more expressed in gonidia $\left(\log _{2}\right.$ (RPM in gonidia/RPM in somatic cell) value $\geqslant 1.0, P$-value $<0.05$, Mann- Whitney $U$ test, one-tailed), whereas 50 miRNAs are more expressed in somatic cells ( $\log _{2}$ (RPM in gonidia/RPM in somatic cell) value $\leqslant-1.0, P$-value $<0.05$, Mann-Whitney $U$ Test, onetailed) (Figure 4A; Table S5 in Supporting Information). Differential expressions of some of the miRNAs in gonidia and somatic cells were further confirmed by Northern blot analysis. Consistent with the deep sequencing results, Vca-miR2, Vca-miR39, Vca-miR82, and Vca-miR101 were mainly detected in somatic cells, whereas Vca-miR35-3p, Vca-miR69, Vca-miR49, and Vca-miR105 were more detected in gonidia (Figure 4B).

To explore the regulatory roles of these differentially expressed miRNAs, we used quantitative RT-PCR to determine the expression levels of some of the miRNA targets. Interestingly, targets of the gonidia-enriched miRNAs, estExt_fgenesh5_synt.C_30114 (Vca-miR19), estExt_ fgenesh4_pg.C_290163 (Vca-miR69), estExt_fgenesh4_ pg.C_10163 (Vca-miR105), estExt_fgenesh4_pg.C_430047 (Vca-miR115), and estExt_fgenesh4_pg.C_450074 (VcamiR117), were expressed at higher levels in somatic cells than that in gonidia; meanwhile, targets of the somatic cellenriched miRNA, estExt_fgenesh4_pg.C_630062 (VcamiR51) was expressed at higher levels in gonidia than that in somatic cell (Figure 4C). The negative correlation of expression levels of miRNAs and their target genes suggests the posttranscriptional regulation of these genes by their cognate miRNAs.

\subsection{A miRNA conserved in Volvox and Chlamydomo- nas}

The identification of miRNAs in Volvox prompted us to examine whether any miRNAs are conserved in Volvox and 
A
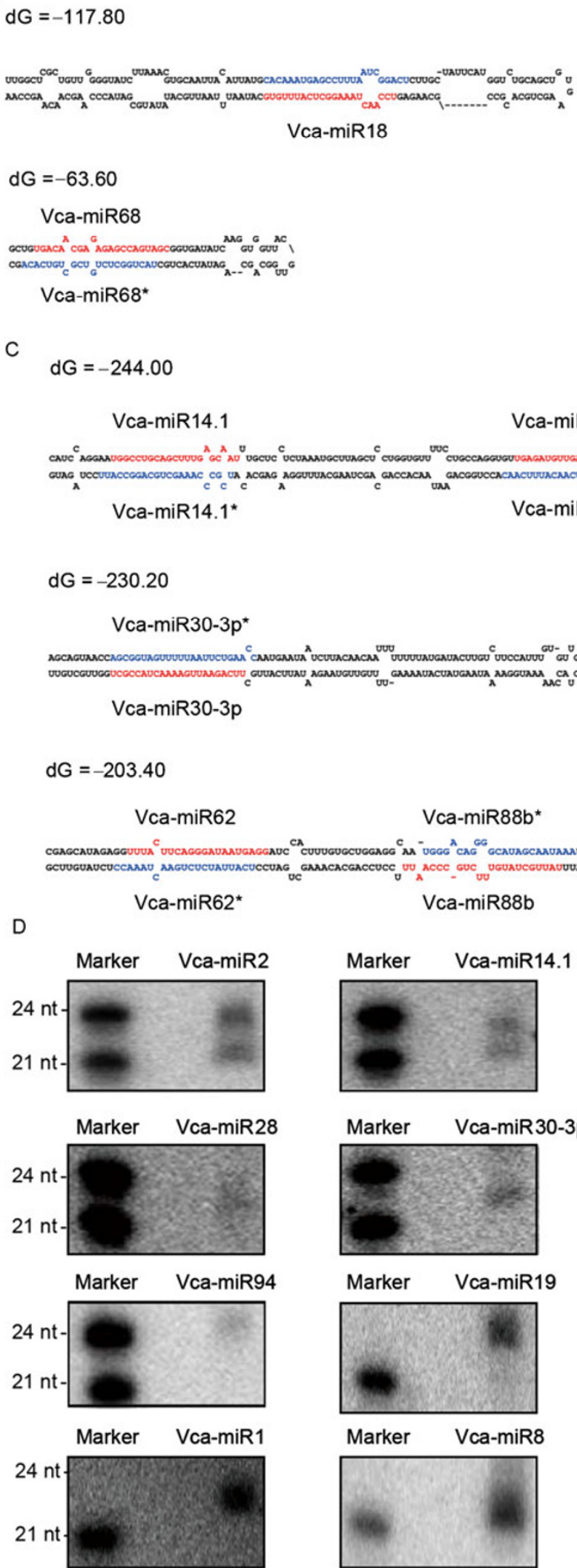

Vca-miR14.2

Vca-miR14.2*

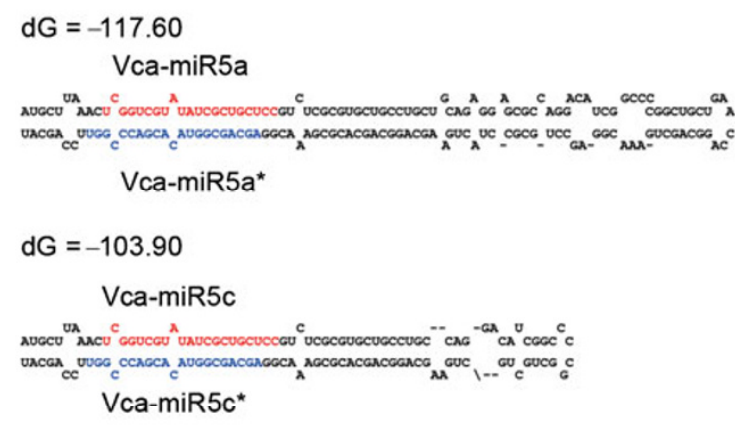

Vca-miR5c* 
EstExt_fgenesh4_pg.C_630062 (Vca-miR51)
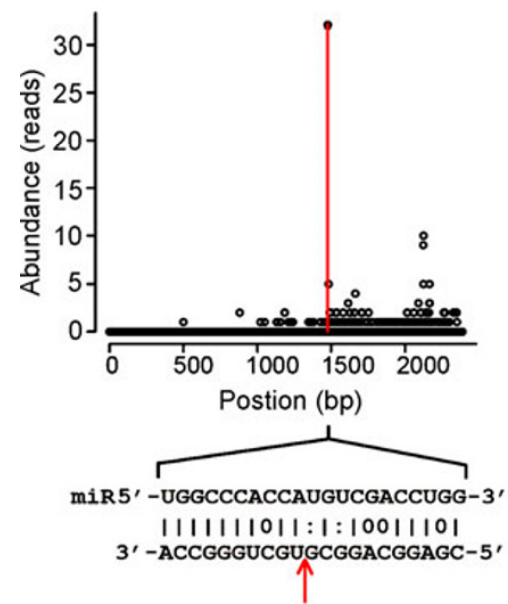

EstExt_fgenesh4_pg.C_430078 (Vca-miR77)

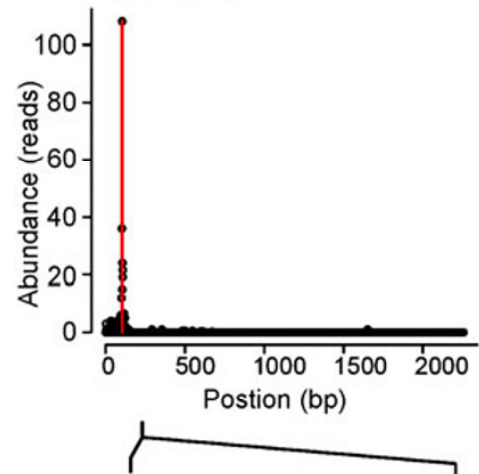

miR 5'-UGGCUCGCACGCGGCCGGCGG-3' 111111111111111111111 3' -ACCGAGCGUGCGCCGGCCGCC-5' EstExt_fgenesh4_pg.C_230029 (Vca-miR39)

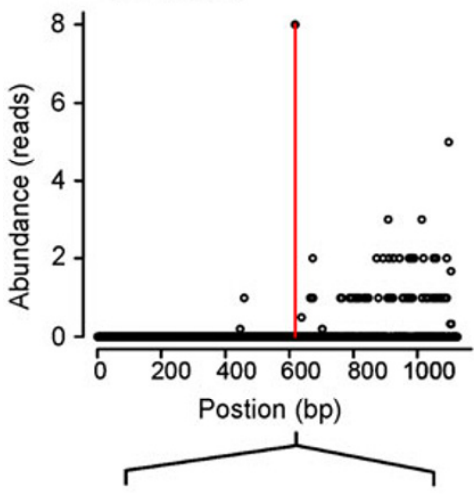

miR 5'-UAUGUAGUAGAagGagaAgCCA-3' : $111111111111: 11111100$ 3' -GUACAUCAUCUUCUUCUUCGAG-5'
EstExt_fgenesh4_pg.C_450074 (Vca-miR117)
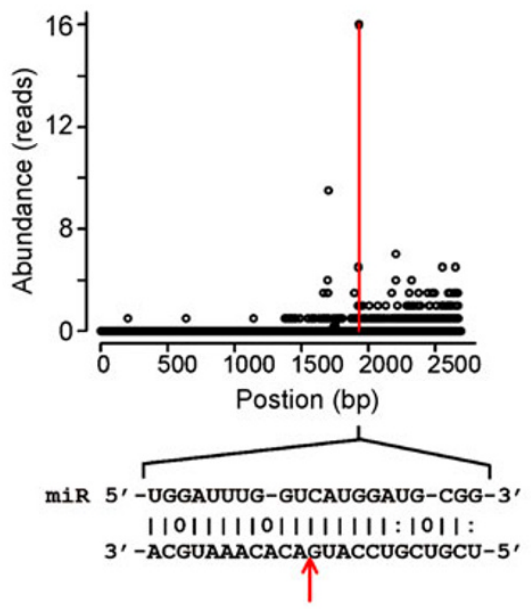

Fgenesh4_pg.C_scaffold_6500010 (Vca-miR93.2)

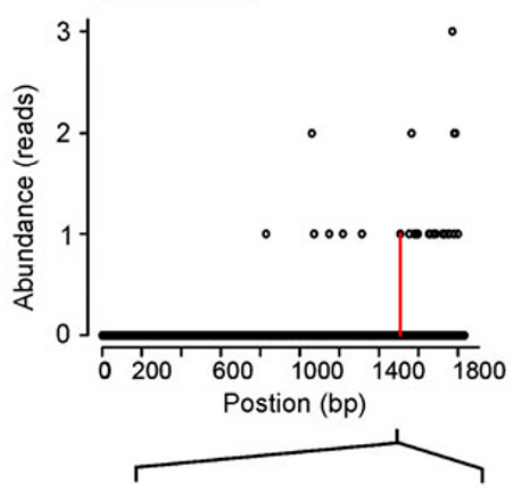

miR 5'-UGCAUCUGCUGCUGCACCUGC-3' 011111111111111011111 3' -UCGUAGACGACGACGAGGACG-5'

Fgenesh4_pg.C_scaffold_11000131 (Vca-miR67)
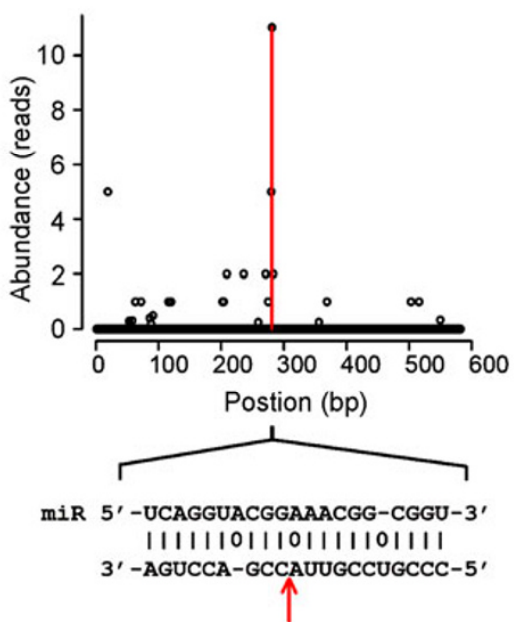

EstExt_Genewise1.C 230182

(Vca-miR103)
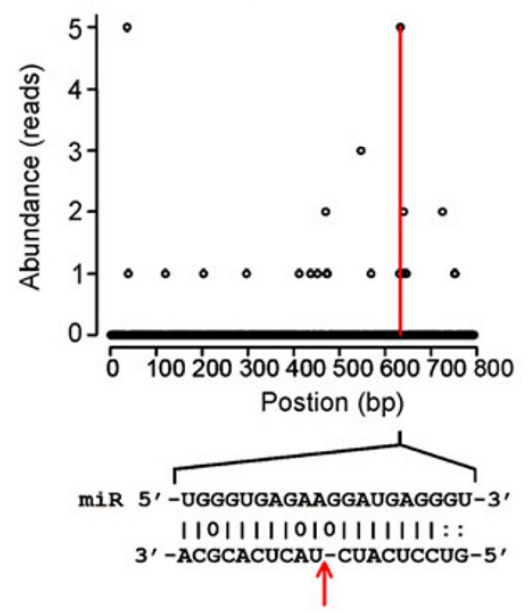

Fgenesh4_pg.C_scaffold_16000045 (Vca-miR63)

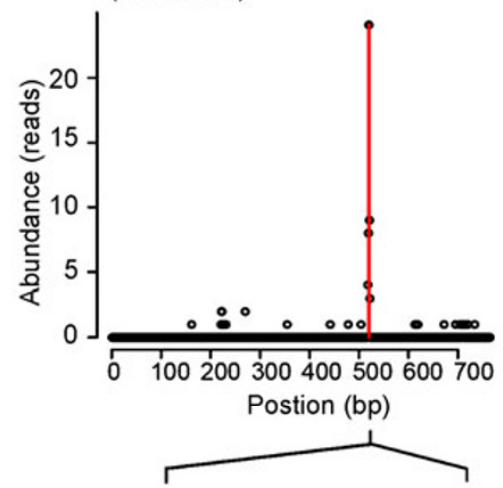

miR 5' -UGACCUACCACGUGGGAGCAGG-3' : $1110111111110111110: 0$ 3' -GCUGUAUGGUGCA-CCUCGGUA-5' $\uparrow$

EstExt_fgenesh4_pg.C_10163 (Vca-miR105)
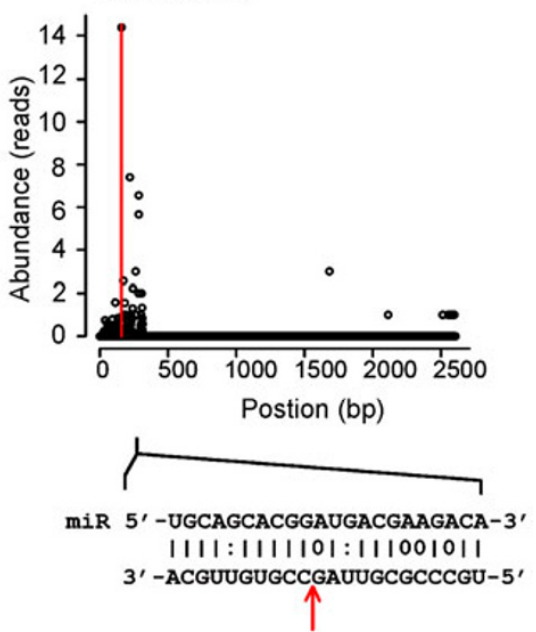

Figure 3 Target plots of Volvox validated miRNA targets. The abundance of each signature from degradome sequencing is plotted and the signatures aligned with \pm 1 positions of miRNA complementary site are combined and shown in red. Alignment of the miRNA and its cognate targets is shown below each plot. Circles indicate non-matched nucleotides and colons indicate G:U wobble pairing. Arrows indicate the predicted cleavage sites. 

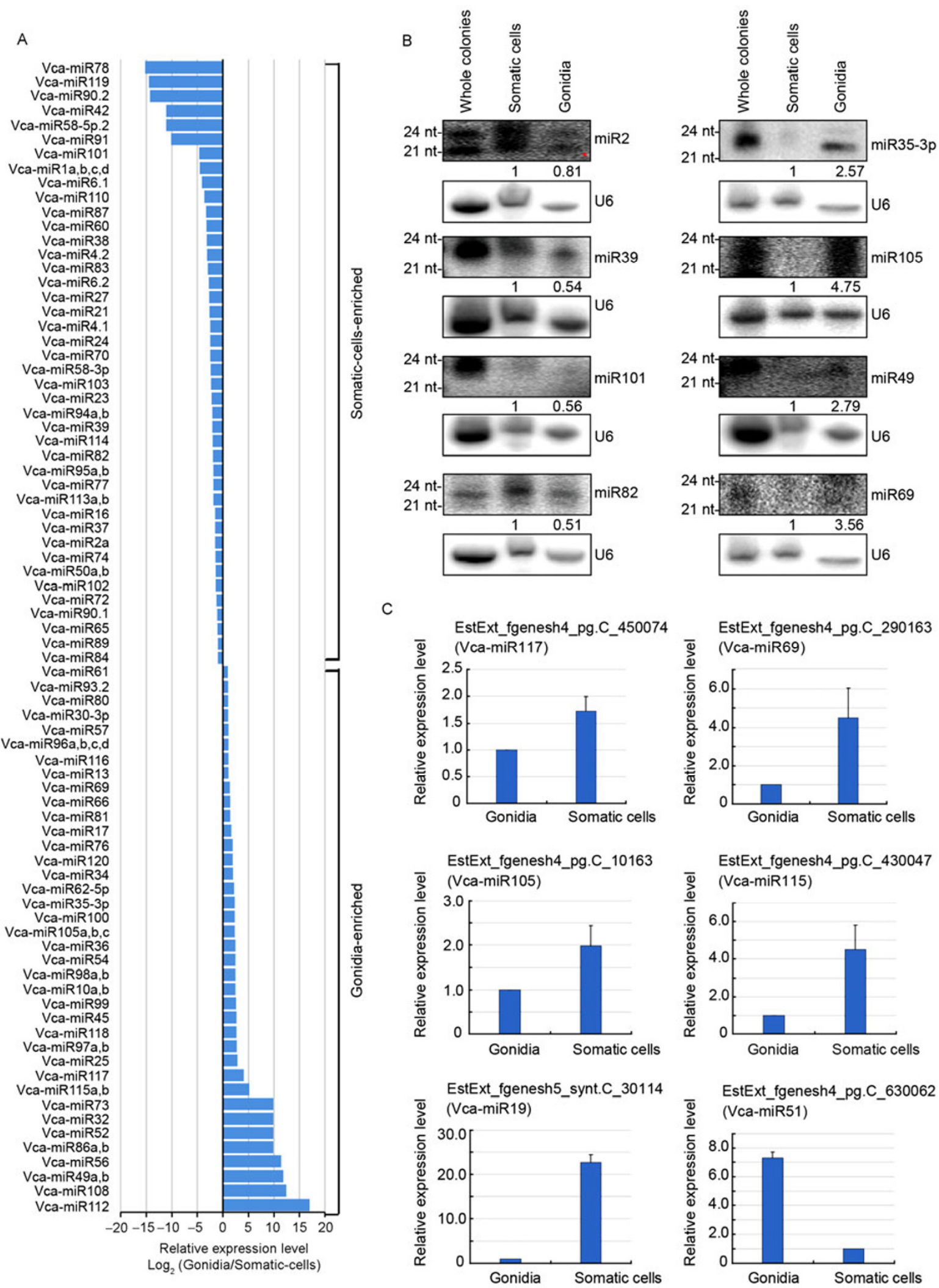

Figure 4 Expression of miRNAs in gonidia and somatic cells. A, A histogram showing differentially expressed miRNAs in gonidia and somatic cells. miRNAs meeting the criteria $\left(P<0.05\right.$ and $\log _{2}(\mathrm{RPM}$ in gonidia/RPM in somatic cell $) \geqslant 1.0$ or $\log _{2}(\mathrm{RPM}$ in gonidia/RPM in somatic cell $\left.) \leqslant-1.0\right)$ are shown. $\mathrm{B}$, Detection of miRNAs in whole colony, gonidia and somatic cells by Northern blot analysis. The relative expression values in gonidia are calculated by comparison with those in somatic cells. C, Detection of miRNA target genes in gonidia and somatic cells. Actin was used as an internal control for data normalization. Error bars indicate SD $(n=3)$. 


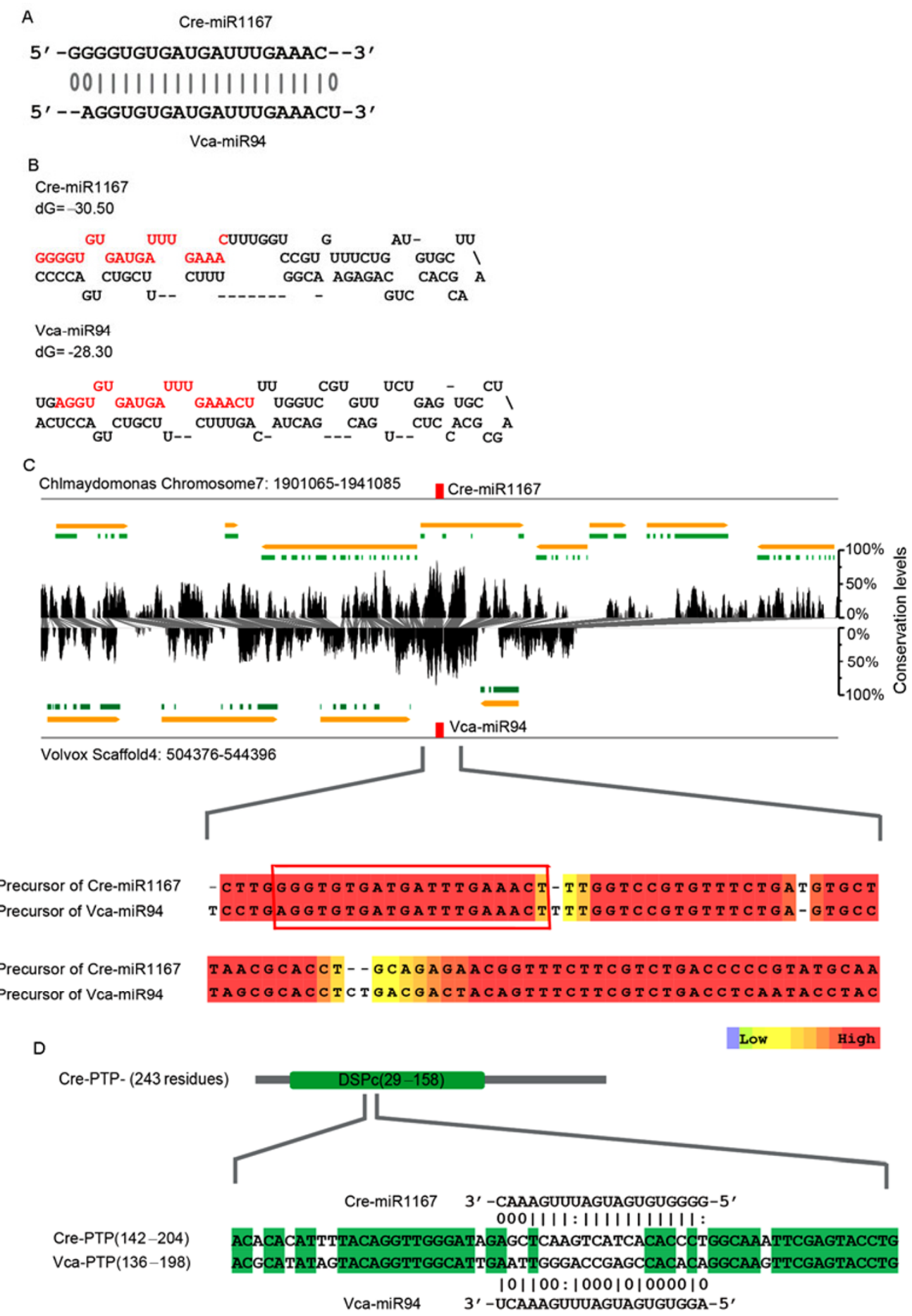

Figure 5 A miRNA conserved in Volvox and Chlamydomonas. A, Alignment of mature sequences of Vca-miR94 and Cre-miR1167. B, Precursors of Vca-miR94 and Cre-miR1167. Sequences corresponding to the mature miRNAs are shown in red. C, Alignment of genomic sequences around Vca-miR94 and Cre-miR1167. $20 \mathrm{~kb}$ up- and down-stream sequences of Cre-miR1167 and Vca-miR94 from Chlamydomonas and Volvox genome were aligned. Yellow chevrons and green boxes represent genes and exons, respectively. Red boxes indicate the mature miRNA sequences. Conservation levels were estimated by the scrolling window method and plotted with black bars. D, Alignment of the nucleotide sequences within DSPc domain of PTP genes of Chlamydomonas and Volvox. Conserved nucleotides are highlighted and the target sites are aligned with miRNAs. Circles indicated non-matched nucleotides and colons indicate G:U wobble pairing.

Chlamydomonas. We searched for homologs of Volvox miRNAs in Chlamydomonas. To our surprise, only one Volvox miRNA (Vca-miR94) shows significant homology to a Chlamydomonas miRNA (Cre-miR1167). This suggests the very high frequency of birth and death of miRNAs in the green algal lineage. 
The mature sequences of Vca-miR94 and Cre-miR1167 differ in two nucleotides (Figure 5A). Mature Vca-miR94 and Cre-miR 1167 are both located in the $5^{\prime}$ arm of the precursors (Figure 5B). In plants, the precursors of orthologous miRNAs and their flanking sequences are very often located in relatively collinear regions with little rearrangement [21]. We thus plotted the alignments of genomic sequences $(\sim 40 \mathrm{~kb})$ surrounding the Vca-miR94 and Cre-miR1167 to evaluate the conservation and divergence in the two species (Figure 5C). The genomic sequences near the precursors of Vca-miR94 and Cre-miR1167 ( 2 kb) show less divergence than the sequences out of this region (Figure 5C). These results suggest that Vca-miR94 and Cre-miR1167 may be evolved from a common ancestor.

In plants, orthologous miRNAs usually regulate orthologous target genes [22]. A predicted target of Cre-miR1167, Cre-ptp, encodes a tyrosine-specific protein phosphatases (PTPase) like protein (Figure 5D), and has a close ortholog (Vca-ptp, similarity $>97 \%$ in protein sequence; Figure S5 in Supporting Information) in Volvox. However, despite the high similarity of overall sequences, the vast majority of the nucleotides in the miRNA binding site in Vca-ptp have been substituted and Vca-ptp can no longer be targeted by Vca-miR94 (Figure 5D). The evolutionary driving force for the loss of miRNA targeting in Vca-ptp is an interesting subject for future investigation.

\section{Conclusion}

In this study, we identified 174 miRNAs that belong to 130 families in Volvox. Two hundred and forty-three target genes were predicted for 60 miRNA families. These miRNA target genes are involved in a variety of biological processes, suggesting the importance of miRNA regulation in the development of growth of Volvox. Our catalog of miRNAs in Volvox as well as the previously identified miRNAs in Chlamydomonas provides a foundation for further biochemical, genetic, and genomic characterization of these RNAs.

This work was supported by the National Natural Science Foundation of China (31225015) and National Basic Research Program of China (2012CB910900) to Qi YiJun.

1 Carthew RW, Sontheimer EJ. Origins and mechanisms of miRNAs and siRNAs. Cell, 2009, 136: 642-655

2 Voinnet O. Origin, biogenesis, and activity of plant microRNAs. Cell, 2009, 136: 669-687

3 Xie Z, Allen E, Fahlgren N, Calamar A, Givan SA, Carrington JC.
Expression of Arabidopsis miRNA genes. Plant Physiol, 2005, 138: 2145-2154

4 Jones-Rhoades MW, Bartel DP, Bartel B. MicroRNAs and their regulatory roles in plants. Annu Rev Plant Biol, 2006, 57: 19-53

5 Zhao T, Li G, Mi S, Li S, Hannon GJ, Wang XJ, Qi Y. A complex system of small RNAs in the unicellular green alga Chlamydomonas reinhardtii. Genes Dev, 2007, 21: 1190-1203

6 Molnar A, Schwach F, Studholme DJ, Thuenemann EC, Baulcombe DC. miRNAs control gene expression in the single-cell alga Chlamydomonas reinhardtii. Nature, 2007, 447: 1126-1129

7 Kirk DL. A twelve-step program for evolving multicellularity and a division of labor. Bioessays, 2005, 27: 299-310

8 Prochnik SE, Umen J, Nedelcu AM, Hallmann A, Miller SM, Nishii I, Ferris P, Kuo A, Mitros T, Fritz-Laylin LK, Hellsten U, Chapman J, Simakov O, Rensing SA, Terry A, Pangilinan J, Kapitonov V, Jurka J, Salamov A, Shapiro H, Schmutz J, Grimwood J, Lindquist E, Lucas S, Grigoriev IV, Schmitt R, Kirk D, Rokhsar DS. Genomic analysis of organismal complexity in the multicellular green alga Volvox carteri. Science, 2010, 329: 223-226

9 Nishii I, Miller SM. Volvox: Simple steps to developmental complexity? Curr Opin Plant Biol, 2010, 13: 646-653

10 Herron MD, Hackett JD, Aylward FO, Michod RE. Triassic origin and early radiation of multicellular volvocine algae. Proc Natl Acad Sci USA, 2009, 106: 3254-3258

11 Kirk DL. Volvox: a Search for the Molecular and Genetic Origins of Multicellularity and Cellular Differentiation. Cambridge: Cambridge University Press, 1998

12 Nematollahi G, Kianianmomeni A, Hallmann A. Quantitative analysis of cell-type specific gene expression in the green alga Volvox carteri. BMC Genomics, 2006, 7: 321

13 Jurka J, Kapitonov VV, Pavlicek A, Klonowski P, Kohany O, Walichiewicz J. Repbase update, a database of eukaryotic repetitive elements. Cytogenet Genome Res, 2005, 110: 462-467

14 Crooks GE, Hon G, Chandonia JM, Brenner SE. Weblogo: a sequence logo generator. Genome Res, 2004, 14: 1188-1190

15 Griffiths-Jones S, Moxon S, Marshall M, Khanna A, Eddy SR, Bateman A. Rfam: annotating non-coding RNAs in complete genomes. Nucleic Acids Res, 2005, 33: D121-124

16 Addo-Quaye C, Eshoo TW, Bartel DP, Axtell MJ. Endogenous siRNA and miRNA targets identified by sequencing of the Arabidopsis degradome. Curr Biol, 2008, 18: 758-762

17 German MA, Pillay M, Jeong DH, Hetawal A, Luo S, Janardhanan P, Kannan V, Rymarquis LA, Nobuta K, German R, De Paoli E, Lu C, Schroth G, Meyers BC, Green PJ. Global identification of microRNA-target RNA pairs by parallel analysis of RNA ends. Nat Biotechnol, 2008, 26: 941-946

18 Addo-Quaye C, Miller W, Axtell MJ. Cleaveland: a pipeline for using degradome data to find cleaved small RNA targets. Bioinformatics, 2009, 25: 130-131

19 Parizotto EA, Dunoyer P, Rahm N, Himber C, Voinnet O. In vivo investigation of the transcription, processing, endonucleolytic activity, and functional relevance of the spatial distribution of a plant miRNA. Genes Dev, 2004, 18: 2237-2242

20 Valoczi A, Varallyay E, Kauppinen S, Burgyan J, Havelda Z. Spatio-temporal accumulation of microRNAs is highly coordinated in developing plant tissues. Plant J, 2006, 47: 140-151

21 Fahlgren N, Jogdeo S, Kasschau KD, Sullivan CM, Chapman EJ, Laubinger S, Smith LM, Dasenko M, Givan SA, Weigel D, Carrington JC. microRNA gene evolution in Arabidopsis lyrata and Arabidopsis thaliana. Plant Cell, 2010, 22: 1074-1089

22 Axtell MJ, Bartel DP. Antiquity of microRNAs and their targets in land plants. Plant Cell, 2005, 17: 1658-1673

Open Access This article is distributed under the terms of the Creative Commons Attribution License which permits any use, distribution, and reproduction in any medium, provided the original author(s) and source are credited. 


\section{Supporting Information}

Table S1 Primers and probes

Table S2 Category of Volvox small RNAs

Tabel S3 Annoataed miRNAs in Volvox

Table S4 Prediction and verification of Volvox miRNA targets

Table S5 Differential expression of Volvox miRNA in gonidia and somatic cells

Dataset S1 Putative precursors of miRNAs in Volvox

Figure S1-S4 Target plots of Volvox validated miRNA targets. The abundance of each signature from degradome sequencing is plotted and the signatures aligned with \pm 1 positions of miRNA complementary site are combined and shown in red. Alignment of the miRNA and its cognate targets is shown below each plot. Circles indicate non-matched nucleotides and colons indicate G:U wobble pairing. Arrows indicate the predicted cleavage sites.

Figure S5 Alignment of Cre-PTP and Vca-PTP protein sequences.

The supporting information is available online at life.scichina.com and link.springer.com. The supporting materials are published as submitted, without typesetting or editing. The responsibility for scientific accuracy and content remains entirely with the authors. 